\title{
As relações universidade-empresa-governo: Um estudo sobre a Universidade Estadual de Campinas \\ (Unicamp *
}

\author{
Sandra Brisolla**, Solange Corder*** \\ Erasmo Gomes ${ }^{* * * *}$, e Débora Mello****
}

RESUMO:Trabalhos realizados atualmente sobre o tema relações universidade e sociedade propõem que estamos vivenciando uma Segunda Revolução Acadêmica, caracterizada pela agregação da função de desenvolvimento econômico regional e local, às já clássicas atividades de pesquisa e ensino desempenhadas pela universidade. Adicionalmente, alguns autores estão desenvolvendo o modelo de Hélice Tripla, uma proposta intermediária entre o livre mercado e o planejamento centralizado, que procura ultrapassar as limitações dos modelos baseados numa visão linear do processo de inovação. Este trabalho é baseado numa pesquisa sobre as relações da Unicamp e seus parceiros externos realizada no período 19951997. Tem por objetivo avaliar a intensidade dessas transformações no caso brasileiro, tomando por objeto de estudo a Unicamp, que foi implantada no início dos anos 60 e que visava fornecer apoio ao processo de industrialização do país.

O trabalho foi desenvolvido em duas etapas. Na primeira foram avaliados 732 contratos realizados no período 1982-1995 e gerenciados

* Este paper sintetiza os resultados do projeto de pesquisa "Universidade-empresa: Ciência e tecnologia", iniciado em agosto de 1995, que foi coordenado pela professora Sandra de Negraes Brisolla. Este projeto era componente de um programa de pesquisa implantado e gerenciado pelo Centro de Estudos Educação e Sociedade (Cedes). Os autores agradecem o auxílio financeiro prestado pelas agências de fomento Finep e CNPq.

** Professsora doutora do Departamento de Política Científica e Tecnológica da Unicamp.

E-mail:sandra@npct.ige.unicamp.br

*** Mestre em Política Científica e Tecnológica pela Unicamp. Fone: (019) 788-8301 e Fax: (019) 289.1772

**** Doutorando em Política Científica e Tecnológica pela Unicamp. E-mail: erasmo@ige.unicamp.br

**** Doutoranda em Política Científica e Tecnológica pela Unicamp. E-mail: deboramello@ibm.net 
pela Fundação de Desenvolvimento da Unicamp. As principais informações obtidas nessa fase foram: freqüência, duração média, valor médio e participação no valor total - por categoria de financiador e por período de tempo. Numa segunda etapa, extraiu-se uma amostra de $25 \%$ do total, resultando em 116 contratos, o que conduziu à realização de 57 entrevistas com professores. Isso permitiu identificar a natureza dos contratos executados em cooperação, o perfil da interação, os seus impactos, as tendências, bem como realizar uma avaliação dos mecanismos institucionais de interação.

Palavras-chave: Unicamp, universidade, empresa, contratos, interação

\section{Introdução}

Os trabalhos realizados atualmente sobre as relações universidade e sociedade têm apontado para a emergência de uma Segunda Revolução Acadêmica. ${ }^{1}$ Para Etzkowitz e Webster, as universidades, além de suas clássicas atividades de ensino e pesquisa, estão, crescentemente, incorporando uma terceira atividade: a atuação em desenvolvimento econômico local e regional. Estes autores sustentam que, no decorrer da Segunda Revolução, está sendo elaborado um novo contrato social entre universidade e sociedade, no qual o financiamento público para a universidade está condicionado à sua contribuição direta para a economia. ${ }^{2}$

Para esses autores, é também possível que as universidades, ao adicionar desenvolvimento econômico às suas atividades anteriores, venham a assumir novos formatos, tal como ocorreu com as instituições de ensino ao incorporar atividades de pesquisa (Etzkowitz 1994). Esse novo formato sugere indagações sobre o futuro da universidade, porém defende-se que uma maior participação da universidade no desenvolvimento local poderá auxiliar no fortalecimento da mesma. Ou, como afirma Etzkowitz (1994, p. 151), pode "transformar-se num processo de renascimento".

As circunstâncias econômicas confrontadas por empresas, universidade e Estado, nos anos 90, são muito diferentes daquelas que prevaleceram nos anos 70 e na primeira metade dos anos 80 , segundo o argumento desenvolvido pelos autores. Para as universidades, o desafio é responder à queda de recursos, tornarem-se seletivas e redefinirem seus objetivos estratégicos. Isto significa a necessidade de reestruturar a univer- 
sidade para o novo ambiente, incluindo-se a criação de novas estruturas organizacionais voltadas para o fortalecimento da atuação em redes, da realização de franchising e da comercialização dos resultados de sua pesquisa e do aumento da oferta de treinamento (Webster 1994a).

No entanto, a tese da Segunda Revolução não recebe uma acolhida unâmine. Peters (1987) defende que o que acompanhamos atualmente é a retomada de laços que foram interrompidos durante o período do pósguerra, quando, baseando-se nas proposições de Vannevar Bush, houve uma intensificação do financiamento governamental à pesquisa. Peters reconhece que tem havido inovações organizacionais na última década, mas acredita que o sistema acadêmico não será radicalmente modificado em suas características e funções básicas: haverá uma acomodação. O exemplo clássico utilizado para ilustrar essa tese é o MIT - Massachussets Institute of Technology. ${ }^{3}$

De uma maneira mais geral, podemos identificar as seguintes razões de estímulo à aproximação entre universidade e empresa: o aumento crescente dos custos de pesquisa e desenvolvimento, tanto para os departamentos de P\&D das empresas industriais, como da pesquisa acadêmica; a diminuição dos recursos governamentais para a pesquisa universitária, associada às mudanças nas políticas governamentais, estimulou os pesquisadores a procurar novas fontes de apoio; a emergência, nos anos 80 , de um novo paradigma científico que diminuiu a distância entre inovação e aplicação tecnológica; a disseminação da busca de novas formas organizacionais para aproximar universidades e empresas, em razão da divulgação dos resultados alcançados por universidades líderes; a necessidade de maior interdisciplinaridade e da adoção de um enfoque globalizado para as soluções dos problemas industriais, que tem conduzido à intensificação da colaboração entre diferentes agentes econômicos e à estruturação de redes e consórcios (Etzkowitz 1989; OCDE 1992; Callon 1992; Leydesdorff e Etzkowitz 1997). Ou seja, "sente-se a necessidade de relações multifacetadas entre organizações, para levar adiante inovações e trazer novos produtos para o mercado no ambiente de competição internacional acirrada dos anos 90" (Etzkowitz e Leydesdorff 1997, p. 4)

Os estudos procuram destacar que é necessário avaliar se tais alterações estão relacionadas a mudanças macroeconômicas mais profundas no ambiente de inovação ou se são, apenas, estratégias de sobrevivência empregadas por empresas, universidades e agências governamentais durante períodos prolongados de instabilidade social e econômica (Webster 1994a, p. 73). 
Todavia, a maior aproximação entre universidade e seu entorno não é uniforme: ela varia segundo áreas do conhecimento e de acordo com a estrutura de pesquisa dos países. Assim, nos Estados Unidos, áreas como química, engenharia, administração e geologia mantiveram vínculos históricos com as empresas em seu setor (Etzkowitz e Peters 1991). Na Alemanha, a atuação conjunta da universidade e da indústria química é bastante destacada. O que mudou nos anos 80 , com a emergência da biotecnologia, é que algumas das áreas que mantinham certo afastamento das atividades empresariais passaram a ser o centro das atenções. Este é o caso da biologia, da bioquímica e de outras áreas correlatas.

Conhecer a estrutura de pesquisa é essencial, dado que a identificação das universidades como o locus da pesquisa básica varia muito entre países. Em alguns deles, as universidades realizam grande parte da pesquisa básica; em outros, universidades e institutos participam igualmente no processo de inovação. E, finalmente, em outros países, é expressiva a participação das empresas privadas em pesquisa conjunta e/ou nos contratos cooperativos. Além disso, o modelo de universidade que incorpora desenvolvimento econômico regional em suas funções não é universalmente aceito. Até mesmo nos Estados Unidos, a Segunda Revolução Acadêmica não afetou as universidades de maneira homogênea (Nelson e Rosenberg 1993; Leydesdorff e Etzkowitz 1997).

Se a organização da pesquisa destaca especificidades entre diferentes países, a criação de mecanismos de interação universidade-empresa indica uma certa homogeneidade entre eles. Em diferentes regiões encontram-se acordos de cooperação, criação de empresas para buscar capital de risco, estabelecimento de trabalhos de consultoria, realização de contratos de pesquisa cooperativa, implantação de parques tecnológicos e participação de professores nos boards das empresas e vice-versa. ${ }^{4}$

Por outro lado, a intensificação dessas relações tem dado origem a diversos conflitos: a compatibilização de tarefas do professor que continua suas atividades na universidade após criar uma empresa ou fazer parte do board de uma empresa; a participação acionária da universidade nas novas empresas; o redirecionamento da pós-graduação para atender aos interesses de empresas e muitos outros. Outro ponto polêmico da cooperação é que as universidades têm promovido acesso de empresas estrangeiras aos resultados de pesquisa, para prejuízo das empresas locais, como observado no caso americano (Mowery e Rosenberg 1993).

No entanto, uma maior aproximação entre universidade e setor empresarial não significa, segundo esses autores, afastamento do Estado. 
Sugere-se um modelo que viabilize a formação de um padrão espiral de ligações nos vários estágios do processo de inovação e que permita meIhor compreender as relações que se estabelecem nas três esferas institucionais - universidade, empresa e governo -, que vêm, crescentemente, atuando em conjunto. Esse modelo, denominado Hélice Tripla, é apresentado como uma proposta intermediária entre o livre mercado e o planejamento centralizado, pois enfatiza a soma das ações conjuntas dentro e entre as esferas institucionais (Etzkowitz e Brisolla forthcoming; Etzkowitz e Leydesdorff 1997). A proposta é que "o crescimento econômico futuro é dependente não apenas de um novo ciclo de inovações, mas de uma nova estrutura para a inovação que ligue a pesquisa básica e a aplicada de forma cada vez mais próxima" (Etzkowitz 1994, p. 141).

Se, para os países centrais, a discussão sobre uma possível nova Revolução Acadêmica faz sentido, dado, entre outros fatores, ao fato de que as universidades destes países já passaram há muito pela fase de incorporação da pesquisa nas funções da universidade, no caso latinoamericano devem ser destacadas algumas especificidades. Por exemplo, em muitos países ou regiões ainda é adotado o modelo de universidade de ensino; não há pesquisa.

Sendo assim, na América Latina, a contribuição mais expressiva esteve dirigida à formação de recursos humanos e os laços de cooperação - quando existiram - ocorreram com as empresas estatais da região, que representavam os setores tecnologicamente mais avançados e com melhor dotação de recursos humanos, até recentemente (Vessuri 1994). O envolvimento de empresas multinacionais e das pequenas e médias empresas foi mais restrito; seja porque, no primeiro caso, a maior parte das tecnologias era importada, havendo concentração na adaptação de produtos e técnicas às condições locais ou devido ao fato de que as pequenas e médias empresas conferiam pequena valorização à variável tecnológica na elaboração de estratégias de crescimento ou consolidação (Sutz 1994).

No caso brasileiro, o aproveitamento do potencial científico e tecnológico das universidades por parte das empresas ainda se encontra num estágio muito incipiente, segundo a avaliação de Velho (1995). E no ambiente marcado pelo neoliberalismo dos anos 90, Plonski (1990 e 1995) defende que arranjos de cooperação entre universidades/institutos e empresas ocorreriam imediatamente, visto estarem sendo solicitadas a apresentar respostas mais rápidas aos desafios das empresas. Por outro lado, Dagnino e Velho (1995) não acreditam na intensificação das relações universidade-empresa nos moldes vistos em países desenvolvidos. Eles 
enfatizam que as universidades - com base neste estudo de caso da Universidade Estadual de Campinas - ainda vêm sendo demandadas para atividades de adaptação de tecnologias importadas para as condições locais, trabalhos de rotina, atividades do tipo trouble shooting e consultorias.

De uma maneira geral, pode-se afirmar que as avaliações sobre as relações universidade-empresa não vêm sendo objeto de reflexão intensa pelos pesquisadores desta região. A maioria dos estudos realizados confere maior destaque às questões pertinentes à área de gestão descrição dos mecanismos e estratégias para intensificação dos laços de cooperação; faltam estudos sobre as barreiras estruturais e institucionais desses países, conforme Brisolla 1995 e Sutz 1994.

O presente estudo pretende contribuir para ampliar a compreensão da problemática envolvendo a interação entre universidade e setor empresarial no Brasil, considerando as condições estruturais locais. Ele foi baseado em uma pesquisa realizada na Universidade Estadual de Campinas (Unicamp), no período de julho de 1995 a julho de 1997. A escolha dessa universidade justifica-se por ser um campo privilegiado de pesquisa, pela sua dimensão e importância na formação de recursos humanos qualificados e pela sua capacitação e contribuição científica no cenário latino-americano. ${ }^{5}$ Outro fator relevante, que justifica a escolha, está relacionado ao propósito de criação desta universidade: ela foi fundada em 1966 com opção pela pesquisa aplicada, pela pós-graduação e por uma vinculação com o setor privado. $\mathrm{Ou}$ seja, seu projeto institucional contemplava explicitamente a disposição em contribuir para o desenvolvimento industrial, interagir com a área empresarial e participar da resolução das questões tecnológicas do país.

O presente estudo pretende identificar a dinâmica da interação, os fatores que contribuem para sua promoção e o papel dos mecanismos de interação universidade-empresa criados pela universidade. Essa pesquisa também pretende contribuir para a reflexão sobre a tese da Segunda Revolução Acadêmica e para o modelo da Hélice Tripla. Este paper está organizado em três itens. No primeiro, apresenta-se a metodologia; no segundo, os resultados obtidos e no terceiro, as considerações finais.

\section{A organização do trabalho: Procedimentos metodológicos}

Numa primeira etapa, buscou-se coletar informações sobre os contratos realizados em cooperação com o setor empresarial. ${ }^{6}$ Utilizou- 
se a base de dados da Fundação de Desenvolvimento da Unicamp Funcamp -, que é a encarregada do gerenciamento administrativo da maioria dos contratos da universidade. Os dados foram coletados para o intervalo 1982-1995, pois para períodos anteriores não havia arquivos disponíveis. Foram avaliados 732 contratos, sendo $46 \%$ relativos às engenharias, $27,2 \%$ à área de ciências exatas, $21,5 \%$ às ciências biológicas e da saúde e $4,4 \%$ à administração da universidade. ${ }^{7}$

No levantamento dos dados foram considerados objetos de estudo os contratos referentes à realização de projetos de pesquisa, ao desenvolvimento tecnológico, à prestação de serviços, ao apoio financeiro à estruturação de cursos de pós-graduação, à realização de seminários, simpósios e reuniões técnico-científicas.

Os financiadores dos contratos foram classificados em nove categorias: empresa privada; empresa estatal; agência de financiamento à pesquisa; administração pública federal; administração pública estadual; administração pública municipal; instituição pública de P\&D; instituição internacional e, por último, universidades e organizações não-governamentais nacionais.

Os contratos foram analisados segundo a freqüência, a duração média, o volume de recursos e o valor médio, por áreas do conhecimento, os períodos de tempo e a categoria do financiador. Os valores dos contratos foram atualizados a preços do mês de dezembro de 1995.

Numa segunda etapa, extraiu-se uma amostra de cerca de $25 \%$ dos contratos realizados com o setor empresarial, o que representou 116 contratos e conduziu à realização de 57 entrevistas com professores-coordenadores dos contratos selecionados. ${ }^{8}$ Em relação ao total de unidades da Unicamp, a referida amostra engloba $73,7 \%$ das faculdades e institutos e $25 \%$ dos núcleos de pesquisa interdisciplinar. Foi utilizado um roteiro semi-estruturado composto por 29 questões agrupadas em quatro blocos: caracterização e avaliação dos contratos, perfil, motivações e tendências da interação. As informações coletadas durante as entrevistas foram organizadas em um banco de dados.

Foram realizadas, ainda, entrevistas adicionais abertas com responsáveis pelos mecanismos institucionais de enlace da Unicamp (Escritório de Transferência de Tecnologia, Centro de Incentivo à Parceria Empresarial e Centro de Tecnologia) ${ }^{9}$ e com o diretor do Instituto Universidade-Empresa, que não é vinculado à Unicamp, mas vem sendo utilizado por professores para facilitar suas relações com as empresas. 
Os contratos em questão não encerram todo o relacionamento da Unicamp com agentes externos. No âmbito da estrutura administrativa da universidade existem outras instâncias dirigidas a essa finalidade, como a Diretoria Geral da Administração - DGA. No entanto, os contratos tramitados através da DGA referem-se, basicamente, a repasse de recursos financeiros de bolsas de pós-graduação e outros auxílios, não sendo encontrados acordos de cooperação entre a Unicamp e o setor empresarial. Adicionalmente, escolheu-se a Funcamp como base para esses estudos, dado que outras fundações que atuam na Unicamp não dispõem de arquivos de dados que cobrem períodos mais longos ou, então, são muito específicas (atendem apenas uma unidade), e, nesse trabalho, interessava avaliar as relações da Unicamp e não de uma unidade em particular.

\section{Resultados da Pesquisa}

\section{Evolução dos contratos por período de tempo}

Para estudar a evolução ao longo do tempo, o período total foi separado em três períodos de cinco anos cada, sendo que ao primeiro período foi somada a média aritmética dos contratos do período, para uniformização. Acompanhando a tendência de recuperação econômica a partir do Plano Cruzado em 1986, cresce expressivamente (240\%) o número de contratos do quinquênio 1981-85 para o quinquênio 1986-90. Como resultado de um maior dinamismo, o montante dos recursos captados cresce cerca de 50\% de 81-85 para 86-90 (v. Quadro 1). Destaque-se que o aumento do número de contratos se deve principalmente a mais contratos com empresas privadas, cujo valor médio é inferior ao dos celebrados com empresas públicas ou com as agências. A diferença de composição por categoria de financiador vai produzir um portfolio de contratos com valor médio $20 \%$ inferior em $86 / 90$ em relação ao período anterior.

O período subsequente corresponde ao primeiro quinquênio dos anos 90, depois das medidas econômicas liberalizantes do governo Collor. O significado da globalização para os contratos entre a Universidade e o meio externo é perceptível pelo resultado altamente negativo tanto sobre o número como sobre o valor médio dos contratos intermediados pela Funcamp. Além de acentuar-se (de 20\% entre 81-85 e 86-90 para 43\% de redução entre $86-90$ e 91-95) a queda no valor médio, verifica-se uma 
queda de aproximadamente $20 \%$ no número dos contratos. (V. Quadro 1). $O$ volume total de recursos captados na primeira metade dos anos 90 equivale a $60 \%$ do valor conseguido no primeiro período analisado e a $40 \%$ do obtido no segundo período. Esse resultado resulta de três processos:

1) A redução de recursos públicos para pesquisa, principalmente por parte da FINEP, que era responsável pela concessão de financiamento a projetos de maior valor médio, mas também do CNPq, que reduziu ao mínimo a concessão de auxílios e aumentou em algumas vezes os recursos para bolsas de pós-graduação. A FAPESP substituiu em parte essa carência, mas para projetos destinados a pesquisa básica e de menor valor médio;

2) A drástica diminuição dos contratos com as empresas públicas, devido à extinção da reserva de mercado, à abertura econômica e ao processo de privatização. Essas empresas antes eram as principais parceiras da pesquisa conjunta da universidade, em projetos caracterizados pela sua maior duração (quando comparados com a cooperação com as empresas privadas) e maior valor médio.

3) A abertura econômica não produziu, como era esperado pela proposta néo-liberal, um maior investimento em pesquisa e desenvolvimento por parte das empresas privadas, e sim o seu contrário. Empresas estrangeiras de maior porte, como a Rhodia e a IBM, reduziram seus recursos para $P \& D$, e muitas das nacionais passaram a importar em lugar de produzir internamente (processo este muito semelhante ao ocorrido na Argentina no passado, com as medidas tomadas por Martinez de Hoz).

Quadro1 - Avaliação de contratos por períodos de tempo

\begin{tabular}{|c|r|r|r|}
\hline Período & Freqüência $^{(\mathrm{b})}$ & \multicolumn{1}{|c|}{$\begin{array}{c}\text { Valor médio } \\
(\mathrm{R} \$ \text { mil) }\end{array}$} & $\begin{array}{c}\text { Volume Total } \\
\text { (R\$ milhões) }\end{array}$ \\
\hline 1981 a $1985^{(\mathrm{a})}$ & 171 & 470,0 & 76,9 \\
\hline 1986 a 1990 & 328 & 380,0 & 112,5 \\
\hline 1991 a 1995 & 264 & 217,0 & 48,5 \\
\hline
\end{tabular}

Fonte: Funcamp - Dados coletados e organizados pelos autores.

Nota: Os totais de contratos em cada coluna não são idênticos, pois havia missing values para alguns contratos. O valor do real é praticamente equivalente ao valor do dólar americano em dez. 1995.

(a) O valor de 1981 foi calculado como equivalente à média do período de 1982-1985 e adicionado ao total do período 1982-1985 para permitir a uniformização do tamanho dos períodos.

(b) Atualização de ativos - Índice Geral de Preços - FGV. Base: dezembro/1995. 
A reestruturação produtiva e organizacional das empresas em busca da competitividade e produtividade é um processo ainda muito tímido, conforme indicam as pesquisas recentes (V. Coutinho \& Ferraz, 1994).

\section{Composição dos contratos segundo}

\section{a categoria do financiador}

Do total de 732 contratos, $27,5 \%$, foram firmados com agências de financiamento, $26,6 \%$, com empresas privadas e $22,4 \%$ com empresas estatais. Essas três categorias respondem por mais de três quartos do número de projetos intermediados pela Funcamp. Visto por outro lado, quase $70 \%$ dos contratos são financiados por instituições públicas (somando agências, administração pública federal, estadual e municipal e os institutos públicos de pesquisa).

O maior valor médio dos projetos é financiado pelas agências, com $\mathrm{R} \$ 588$ mil, aproximadamente. Na sequência encontram-se as empresas estatais, com $R \$ 316$ mil, aproximadamente. Os contratos de menor valor são celebrados com os institutos públicos de pesquisa, com $\mathrm{R} \$ 27$ mil em média. As empresas privadas ocupam a penúltima posição entre as categorias de financiadores, com um valor médio de $R \$$ 82,5 mil por contrato ( $v$. Quadro 2). Pode-se, assim, entender o impacto representado pela redução dos contratos com as empresas estatais e

Quadro 2 - Perfil dos Contatos, Segundo a Categoria do Financiador, no Periodo 1981-1995

\begin{tabular}{|l|r|r|r|r|}
\hline \multicolumn{1}{|c|}{ Categoria do financiador } & Freqüência & $\begin{array}{c}\text { Duração média } \\
\text { (meses) }\end{array}$ & $\begin{array}{c}\text { Valor médio } \\
(\mathrm{R} \$ \text { mil) }\end{array}$ & $\begin{array}{c}\text { Volume recursos } \\
\text { (R\$ milhões) }\end{array}$ \\
\hline Agência de financiamento & 201 & 30,7 & 588,0 & 118,2 \\
\hline Empresa privada & 195 & 16,3 & 82,6 & 16,1 \\
\hline Empresa estatal & 164 & 21,1 & 315,9 & 51,8 \\
\hline Inst. Públ. P\&D & 55 & 13,0 & 27,2 & 1,5 \\
\hline Adm. Públ. Federal & 39 & 12,6 & 102,0 & 34,0 \\
\hline Adm. Públ. estadual & 35 & 12,3 & 277,5 & 9,7 \\
\hline Adm. Públ. Municipal & 15 & 13,8 & 130,6 & 2,0 \\
\hline Instituição internacional & 14 & 32,4 & 148,5 & 2,1 \\
\hline Univ. e ONGs nac. & 14 & 17,4 & 180,0 & 2,5 \\
\hline Total & 732 & 21,0 & 325,0 & 237,9 \\
\hline
\end{tabular}

Fonte: Funcamp - Dados coletados e organizados pelos autores.

Nota: Os totais de contratos em cada coluna não são idênticos, pois havia missing values para alguns contratos.

(a) Atualização de ativos - Índice Geral de Preços - FGV. Base: dezembro 1995. 
as agências (principalmente a FINEP) e sua substituição pelos magros projetos financiados pelas empresas privadas, sobre o volume de recursos dos contratos para a Universidade.

A duração média dos contratos é maior no caso de instituição internacional, com média de 32,5 meses. Mas em termos de recursos financeiros esses convênios são bastante modestos. Geralmente referem-se a apoio institucional de pequena monta, para compra de material de consumo de laboratórios, bolsas de estudos e outras atividades que por sua natureza desenvolvem-se por períodos mais longos. As agências de financiamento contratam projetos por um período médio de 30,7 meses, e as empresas estatais, por 21 meses em média, períodos de contrato bastante elevados. Destaque-se que os contratos com empresas privadas na Unicamp duram em média 16 meses, quando em outras instituições eles dificilmente ultrapassam um ano de duração.

Desprende-se desses dados que, excetuando-se as agências de financiamento, são as empresas estatais as que maiores contribuições realizaram para o financiamento à pesquisa acadêmica. Dados similares foram encontrados por Vessuri (1994). O valor médio dos contratos celebrados com o setor privado, de cerca de 80 mil dólares, apesar de ser sete vezes menor que o valor médio financiado pelas agências de financiamento, não é insignificante. Por essa via normalmente entram financiamentos de rubricas não cobertas pelas agências ou mesmo por contratos com organismos estatais, o que em muitos casos torna possível adquirir materiais e equipamentos mais rapidamente, pagar complementação salarial e contratar bolsistas (Mello \& Velho, 1996). Por outro lado, o valor médio do financiamento pelas agências tendeu a cair no período, com a redução dos recursos da FINEP para a pesquisa acadêmica.

É no volume total de recursos segundo a categoria do financiador que se percebe a importância das agências no financiamento à pesquisa. Elas são responsáveis por um montante de $R \$ 118$ milhões, aproximadamente a metade dos recursos extra-orçamentários que entram via Funcamp para a Universidade. Seguem-se as empresas estatais e a administração pública federal. Do total de recursos externos que ingressam na Universidade por meio da Funcamp, as instituições públicas respondem por $R \$ 217,2$ milhões, contra apenas $R \$ 16$ milhões das empresas privadas.

Uma indicação da redução do valor médio dos contratos com o acréscimo da participação das empresas privadas no total de contratos 
pode ser inferida pelo padrão dos contratos com as empresas privadas: elas representam $26.6 \%$ do número de contratos mas apenas $6,8 \%$ do valor. Este percentual constitui uma evidência da importância do investimento estatal na pesquisa acadêmica, ao mesmo tempo que mostra os limites do financiamento privado a universidades no Brasil. Mesmo nos EUA, o setor privado financia apenas $5,5 \%$ da pesquisa acadêmica $(7 \%$ se excluirmos do cálculo os centros de P\&D universitários financiados pelo governo federal) e isso representa apenas $1,5 \%$ de seus gastos em P\&D; o governo, por sua vez, financia $74 \%$ dos gastos em P\&D nas universidades americanas. Cerca de $97,5 \%$ dos recursos investidos pela indústria em P\&D são, portanto, destinados a seus próprios laboratórios e centros de pesquisa (NSF, 1996).

A situação das empresas localizadas no Brasil é bastante diferente: a maioria das empresas que operam em setores intensivos em tecnologia no país são de capital prioritariamente estrangeiro e estas importam as tecnologias de suas matrizes. As empresas privadas de capital majoritariamente nacional realizam investimentos pouco significativos em P\&D, limitando-se a importar tecnologias.

Avaliação da interação, segundo os participantes

Entrevistas realizadas com professores universitários envolvidos na interação com o setor empresarial mostraram que a grande maioria dos contratos realizados com o setor foi voltada para a pesquisa e/ou o desenvolvimento (68\%), sendo que $41 \%$ foram projetos de $P \& D, 14 \%$ apenas de pesquisa e $13 \%$ de cunho exclusivamente tecnológico.

Atividades de prestação de serviços corresponderam a 19\%, e cursos e treinamentos responderam por apenas $6 \%$ dos contratos. Contratos envolvendo consultorias/assessorias, projetos de engenharia, análises de rotina, informações técnico-científicas, como um todo, representaram apenas $5 \%$.

Informações prestadas pelos entrevistados indicam que, aproximadamente, $90 \%$ dos contratos foram bem-sucedidos. É provável que os contratos tenham sido realmente bem-sucedidos, uma vez que em $57 \%$ dos casos o pesquisador se manteve em contato com a empresa e soube que ela utilizou os resultados do trabalho em suas atividades. Isso nos 
mostra que existe um bom fluxo de comunicação entre a universidade e as empresas.

Adicionalmente, a maioria dos entrevistados, cerca de $88 \%$, considerou que os contratos foram bons ou excelentes. Numa média geral, cada pesquisador apontou pelo menos três fatores positivos por contrato, enquanto apenas um fator negativo foi indicado, sugerindo que a interação tem resultado em boas experiências para os entrevistados.

Os quatro principais fatores positivos apontados foram: recursos humanos adequados, recursos financeiros suficientes, precisão dos objetivos do trabalho contratado com a empresa e bom apoio da empresa. Os aspectos negativos mais apontados vão em sentido simétrico ao apontado acima: deficiente apoio da empresa, imprecisão dos objetivos do trabalho contratado com a empresa e recursos financeiros insuficientes. Assim, parece existir uma identidade entre os fatores tanto em seus aspectos positivos quanto nos negativos.

Em cerca de $55 \%$ dos casos os entrevistados relataram a existência de relações de cooperação envolvendo membros da entidade financiadora e equipes de pesquisadores. Isso foi especialmente verdadeiro para os contratos de P\&D, embora nas prestações de serviço, nas quais era esperada uma baixa interação entre as equipes, relações de cooperação foram também significativas.

E, embora a maior parte dos entrevistados tenha relatado experiências positivas em seus contatos com agentes externos, há ainda diversos problemas a solucionar nas relações entre universidade e seu entorno. Alguns estudos de caso destacam uma certa resistência na introdução da lógica empresarial na academia. No estudo de Castro e Balán (1994b) sobre os Institutos de Física da Unicamp e da USP, os professores entrevistados afirmam que o financiamento da pesquisa não deve ser feito pela empresa, pois os trabalhos solicitados são, na maioria da vezes, de baixo conteúdo científico. Por outro lado, há estudos que revelam as resistências do setor empresarial com relação à universidade. O estudo de Stefanuto (1995) mostra que alguns funcionários da Telebrás hostilizaram o grupo de pesquisadores contratados, gerando várias situações de conflito e atrito direto entre usuários e criadores de um programa.

Para Castro e Balán (1994a), a universidade geralmente tem sido incapaz de estabelecer uma "cooperação virtuosa" com o setor empresarial: os contratos firmados - pela sua condução e, também, pela falta de tempo - não têm estimulado os contratantes a investir em ciência e tecnologia. 
A partir das entrevistas realizadas também podem ser identificadas dificuldades pelo lado das empresas: a) obstáculos ao estabelecimento de parcerias em épocas de crise como a atual e b) dificuldade de comunicação devida à inexistência de interlocutores nas empresas.

\section{A aproximação entre os atores}

Os contatos com o setor empresarial foram, em sua maioria, estabelecidos por iniciativa das empresas. Em cerca de $65 \%$ dos contratos avaliados, a empresa é que procurou o pesquisador para a elaboração do trabalho. Em $23 \%$ dos casos foi o pesquisador quem procurou a empresa. Em menos de 3\% dos contratos houve a presença de um intermediário viabilizando a interação.

Os fatores que têm levado os pesquisadores a interagirem com o setor empresarial, atualmente, são a busca de recursos financeiros indicada em $43,4 \%$ das respostas - e a identificação de temas de pesquisa - que alcançou $40,8 \%$ das respostas. Era previsto que a busca de recursos financeiros fosse apontada como uma das motivações mais importantes, uma vez que nos últimos anos as agências de fomento reduziram os recursos para pesquisa.

Segundo os entrevistados, o critério mais relevante utilizado pelas empresas para identificar um parceiro foi a qualificação da equipe de pesquisa. Esse fator já tinha sido identificado por Castro (1993) e por Perre (1995). Para esses autores, o estabelecimento de relações entre universidade e setor empresarial, bem como sua manutenção, depende fortemente do reconhecimento acadêmico do professor envolvido na interação. No entanto, Rachid (1995) mostra que no caso do Programa de Engenharia de Materiais (Dema) da USP/São Carlos, apesar de seus docentes contarem com elevada qualificação profissional, da existência de laboratórios de P\&D na área de materiais e processos e da proximidade do setor empresarial, o Dema ainda tem papel limitado em seu objetivo inicial de interação com as empresas.

É interessante notar que a informalidade marcou os contatos iniciais. Em mais de $50 \%$ dos contratos estudados, os contatos pessoais informais foram a principal via de acesso utilizada pelos atores - professores ou empresas. Ex-alunos, participação em congressos, organização de workshops, foram mencionados como situações que favoreceram o 
estabelecimento de contatos, não apenas de iniciativa das empresas, mas também, de professores.

Diversos estudos nacionais e internacionais também mencionam a relevância dos contatos pessoais informais, sendo que alguns deles destacam o papel dos alunos de graduação e/ou pós-graduação no estímulo à interação (Dierdonck et al. (1990, p. 560), Stefanuto 1995 e Quirino 1993). Esses estudos confirmam a opinião de alguns pesquisadores de que em boa parte dos casos o desafio que a empresa enfrenta não é estimulante para a pesquisa acadêmica, mas presta-se a trabalhos de consultoria de alunos.

O comportamento observado no caso dos professores da Unicamp mostra que, até o momento, é bastante tímido o desempenho dos mecanismos institucionais de enlace, tais como a Companhia para o Desenvolvimento Tecnológico (Codetec), o Centro de Tecnologia (CT), o Centro de Incentivo à Parceria Empresarial (Cipe) e o Escritório de Transferência de Tecnologia (ETT). Isto é verdadeiro não apenas na Unicamp: Dierdonck et al. (1990, p. 560) afirmam que "não há uma clara definição do papel dos escritórios de transferência na estratégia de pesquisa da universidade, e não há consenso sobre quais tarefas estes devem desempenhar".

Impactos e tendências da interação

Para a quase totalidade dos entrevistados houve alterações significativas em suas atividades em decorrência de contratos que contavam com a participação de atores externos à universidade. A atividade docente tem muito a ganhar com a interação, dado que o docente tem possibilidades de ampliar sua experiência e de repassar esses conhecimentos para os alunos; outras vantagens frequentemente mencionadas foram o aumento de volume de recursos financeiros e a diversificação de suas linhas de pesquisa. Outros aspectos positivos mencionados incluem a maior flexibilidade fornecida pelos recursos dos contratos e a maior cautela que o professor passa a ter na negociação de futuros contratos. Alguns aspectos negativos da interação também foram relatados, dentre eles a redução da oferta de disciplinas ou de publicações, a diminuição da dedicação para com os alunos e o efeito negativo na formação dos alunos, dado que desde o início o aluno volta-se a questões de curto prazo das empresas e não valoriza uma formação mais generalista. 
Para a universidade, o impacto mais mencionado pelos entrevistados foi a maior visibilidade desta junto à sociedade. A manutenção dos trabalhos em cooperação auxilia no processo de validação social da universidade, segundo a interpretação dos entrevistados. $O$ segundo impacto mencionado, para a universidade, foi o aumento de recursos financeiros. É interessante observar que na questão anterior este item foi o terceiro, ou seja, os recursos financeiros foram considerados mais importantes para a universidade como um todo, do que para o professor em particular. Talvez isso reflita o constrangimento do professor em reconhecer a relevância desse fator para sua decisão, o que não ocorre quando o interesse passa a ser coletivo (da universidade). Aproximadamente $23 \%$ dos entrevistados adquiriram novos equipamentos com a interação. Alguns entrevistados apontaram outros impactos positivos, como a ampliação dos grupos de pesquisa e o treinamento de pessoal; o treinamento de alunos e a atualização de disciplinas, propiciados pelos trabalhos realizados em cooperação com empresas. Porém, alguns entrevistados destacaram fatores negativos, como a diminuição da atenção à formação de recursos humanos e a perda da pluralidade na escolha de temas de pesquisa.

A grande maioria dos entrevistados (77\%) acredita que as relações com o setor empresarial tendem a crescer. Empresas estatais foram indicadas como futuros colaboradores apenas por um pequeno número de entrevistados; isto é explicado tanto pela fase de privatizações, como pela perda de dinamismo dos centros de pesquisa diretamente vinculados a essas empresas.

É interessante notar que há uma inversão de ordem de importância entre pesquisa e desenvolvimento e prestação de serviços, bem como maior expressão de cursos e treinamentos na projeção para os próximos anos. É provável que os entrevistados estejam percebendo que a prestação de serviços e os cursos e treinamentos são as atividades que melhor se adaptam à tendência de ampliação da aproximação com outros atores.

Finalmente, observou-se que questões de propriedade intelectual estão sendo pouco abordadas. Ainda que algumas patentes possam resultar da parceria universidade-setor empresarial, a orientação oferecida na UNICAMP parece "pouco expressiva e desatualizada quando comparada a padrões internacionais". Nos contratos analisados, menos de $50 \%$ continham cláusulas relacionadas à proteção de 
propriedade intelectual. É, também, elevado o número de casos em que a propriedade intelectual nunca foi abordada (31\%), seja porque não havia necessidade, seja por falta de atenção ao fato.

\section{Considerações Finais}

O estudo da interação da Unicamp-setor empresarial, nos últimos 15 anos, mostrou que a maioria dos contratos foi considerada exitosa, sendo que foram mais freqüentemente mencionados pelos entrevistados os aspectos positivos do que os negativos.

A presença da Unicamp nessas parcerias, no entanto, não decorre de iniciativa da universidade, mas reflete uma busca dos agentes externos de estabelecer parcerias com a Unicamp. Adicionalmente, os ganhos financeiros da interação têm sido limitados e poucos contratos contêm cláusulas de proteção de direitos de propriedade ou que regulem a distribuição de eventuais ganhos.

Os professores entrevistados mostram certa incerteza em relação ao futuro da interação. As empresas estatais que participaram ativamente no financiamento de alguns grupos de pesquisa estão sendo privatizadas. As empresas privadas demonstram interesse limitado em inovação tecnológica, seja por meio da montagem de seus laboratórios de P\&D ou do financiamento de grupos universitários de pesquisa. Essa falta de interesse das empresas privadas é reforçada pelas políticas governamentais contraditórias implementadas nos últimos anos.

Alguns autores sugerem que o papel da universidade deva ser redefinido. No entanto, outras questões deveriam ser colocadas também: Qual o papel que empresas privadas, privatizadas e estatais desejam desempenhar no processo de inovação? Deve o governo apoiar o processo de inovação, seja fornecendo fundos ou desenhando e implementando políticas?

Existe uma convicção generalizada de que as universidades devem e podem desempenhar um papel muito importante no encurtamento do gap tecnológio que constitui sério obstáculo para o crescimento econômico do País. Mas ainda não há consenso sobre as formas através das quais essas instituiçoes irão lograr uma inserçao positiva no sistema nacional de inovação. As tentativas do governo de incentivar o estreitamento de laços entre universidade e empresa não tiveram até 
hoje os resultados esperados, mas a crescente preocupação das empresas com a competitividade deverá mudar os prognósticos daqui por diante dessa interação, que constitui um recurso importante para essa finalidade. As novas configurações propostas para os sistemas de inovação incorporam redes de interrelação entre empresas, universidades e institutos de pesquisa. Nessas redes, que constituem a base da nova sociedade do conhecimento, a universidade terá necessariamente um papel transcendental.

\section{Notas}

1. A Primeira Revolução Acadêmica ocorreu no século XIX quando à função de ensino foi acrescida a atividade de pesquisa. O modelo de universidade que representa o rompimento com o padrão tradicional de universidade foi a Universidade de Berlim.

2. Ver Etzkowitz (1989); Webster e Etzkowitz (1991); Etzkowitz e Peters (1991); Webster (1994a e b) e Etzkowitz (1994).

3. Esta universidade mantém firmes laços com o setor empresarial desde sua implantação, nos Estados Unidos, na segunda metade do século XIX. Diversas inovações institucionais podem ser localizadas na sua trajetória, desde então: a prática da consultoria, os spin-offs, os centros de pesquisa universitária, os parques tecnológicos, a criação dos escritórios de transferência de tecnologia.

4. Para detalhes ver: Dickson, 1988; Etzkowitz e Peters, 1991; Quintas e outros, 1992 e Gomes, 1995.

5. A Unicamp conta, atualmente, com 53 unidades, sendo 19 faculdades e institutos, 20 centros e núcleos de pesquisa interdisciplinar e 14 centros de serviços. É considerada uma das 3 universidades mais importantes do país e centro de excelência em diversas áreas.

6. Por setor empresarial entende-se empresas privadas e empresas estatais.

7. Não foram incluídas as áreas de ciências humanas, letras, artes e filosofia. Não puderam ser classificados em áreas do conhecimento $0,9 \%$ dos contratos.

8. Para o cálculo da amostra foram somados os contratos realizados em cooperação com empresas privadas, empresas estatais e administrações públicas (ou seja, 448 contratos).

9. Nesse trabalho adotou-se uma distinção entre mecanismos institucionais de enlace e mecanismos institucionais de gerenciamento. Os primeiros, como 
é o caso do Escritório de Transferência de Tecnologia, têm por missão estimular as relações entre universidade e setor empresarial. O segundo tipo é instrumento facilitador da interação. Os mecanismos de enlace são de criação recente (anos 90) e não dispõem de base de dados abrangentes. A base de dados analisada - a Funcamp - enquadra-se no segundo tipo e dispõe de um bom arquivo.

10. No estudo realizado com projetos tecnológicos da Unicamp, em 1989 esta hipótese também é confirmada (V. Brisolla 1990).

11. Ao comparar os $6,8 \%$ dos recursos realizados pelas empresas privadas na Unicamp com os $5,5 \%$ investidos pela indústria na pesquisa universitária americana, deve-se salientar que os dados da Funcamp envolvem não apenas atividades de P\&D, mas também prestação de serviços, treinamento e outros.

\section{The universty-industry-govermnment relationship: A study of the State University of Campinas (Unicamp)}

ABSTRACT: Recent studies on the relationship between university and society have indicated the emergence of a Second Academic Revolution, as to the classical activities of teaching and research, universities are now incorporating local and regional development. Additionally, some authors suggest the Triple Helix concept, an intermediate proposal between free market economy and a centrally planned one, and an alternative for the linear innovation model approach.

The present survey intends to appraise the intensity of these transformations in the Brazilian case, more specifically at a renowned university, Unicamp - established in the early 1960s to perform technological research to support the country's industrialisation process.

First, data from Foundation for the Development of Unicamp were collected for the period 1982-1995. 732 contracts were evaluated. Contracts were analysed as to number, average duration, total funding, and average value, as well as by date and category of the funding agency.

In the second part, a sample of $25 \%$ of the contracts conducted with industry and public administration was selected, a total of 116 contracts leading to 57 interviews with professors-coordinators of the selected contracts. Data collected during this phase were related to characterisation and evaluation of contracts, profile, motivation and interaction tendencies, as well as an appraisal of a liaison office. 


\section{Agradecimentos}

Os autores agradecem os comentários de Léa Velho e Renato Dagnino, professores do Departamento de Política Científica e Tecnológica da Unicamp, e da doutoranda Stela Meneghel, da Faculdade de Educação da Unicamp, embora as opiniões e análises sejam de responsabilidade exclusiva dos autores.

\section{Bibliografia}

BRISOLLA, S.N. "A relação da universidade com o setor produtivo - O caso da Unicamp". Revista de Administração da USP nำ1, jan.-mar. 1990, vol.25, pp. 106-126.

. "Capacitación tecnológica y patrones tecnológicos: Una visión a partir de los países en desarrollo". Redes - Revista de Estudios Sociales de la Ciencia № 5. Buenos Aires, dezembro de 1995, vol. 2, pp. 35-65.

CASTRO, M.H.M. "Estudo dos Centros de Excelência: Departamento de Engenharia Mecânica da Universidade Federal de Santa Catarina." Cadernos de Gestão Tecnológica,no 4. São Paulo, NPGCT/USP, 1993, 49p.

CASTRO, M.H.M. e BALÁN, J. "Universidade versus setor empresarial: A perspectiva e a realidade da universidade - Caso 2: As Faculdades de Engenharia Elétrica da Unicamp e da USP". São Paulo, Nupes, 1994a, 43 p. (Série Documentos de Trabalho, 7/94).

. "Universidade versus setor empresarial: A perspectiva e a realidade da universidade - Caso 1: Os Institutos de Física Gleb Wataghin e o da USP (IF)". São Paulo, Nupes, 1994b, 41 p. (Série Documentos de Trabalho, 7/94).

CALLON, M. "The dynamics of techno-economic networks". In: Coombs, R.; Saviotti, P. e Walsh, V. (orgs.). Technological change and company strategies. Londres: Academic Press, 1992, pp. 72-102.

DAGNINO, R.P. "A universidade e a pesquisa científica e tecnológica". In: Ciência e Cultura. São Paulo, SBPC, junho de 1985, pp. 35-65. (Suplemento).

DAGNINO, R. e VELHO, L. "University-industry-government relations in the perifery: The University of Campinas, Brazil" 1995. (a ser publicado na Minerva). 
DICKSON, D. The new politics of science. Chicago, Univ. of Chicago, 1988, 403p.

DIERDONCK et al. "University-industry relationship: How does the Belgian academic community feel about it?" In: Research Policy, 1990.

ETZKOWITZ, H. "Entrepeneurial science in the academy: A case of transformation of norms". Social Problems no 1. Fev. 1989, vol. 36, pp. 14-29.

. "Academic-industry relations: A sociological paradigm for economic development". In: Leydersdorff, L. e Van den Besslaar, P. Evolutionary economics and chaos theory: New directions in technology studies. Londres, Pinter Publishers, 1994, pp. 139-151.

ETZKOWITZ, H. e BRISOLLA, S. "Failure and Success: The Fate of Industrial Policy in Latin America and South East Asia". Forthcoming in Research Policy.

ETZKOWITZ, H. \& LEYDESDORFF, L. Universities in the Global Knowledge Economy: A Triple Helix of Academic-IndustryGovernment Relations. Londres, Cassell, 1997.

ETZKOWITZ, H. e PETERS, L.S. Profiting from knowledge: Organizational innovations and the revolution of academics norms, 1991.

GOMES, E.J. "A experiência brasileira de pólos tecnológicos: Uma abordagem político-institucional". Campinas, Unicamp/ DPCT, 1995 (dissertação de mestrado).

KECK, O. "The national system for technical innovation in Germany". In: Nelson, R.R. (org.). National Innovation Systems. Nova York, Oxford Universits, 1993. pp.115-157.

LEYDESDORFF, L. e ETZKOWITZ, H. "The future location of research: A triple helix of university-industry-government relations" II. 1997 (Theme paper, Conference New York city, 7-10 january, 1998).

LLOVERA, A. de Sola. "Nadando contra la corriente:I\&D en la universidad" In: VESSURI, Hebe (org.). La academia va al mercado - relaciones de científicos con clientes externos. Caracas, Fondo Editorial Fintec, 1995. pp. 321-346.

MELLO, D. e VELHO, L. "As relações universidade-sociedade: Um novo contrato social?" In: II Jornadas Latinoamericanas de Estudios Sociales de la Ciencia y la Tecnologia. Caracas, 9-11 set. 1996. 
MERCADO, Alexis A. "La contitución del Centro de Investigaciones Químicas, Biológicas y Agrícolas de la Unicamp - el CPQBA". In: Vessuri, Hebe (org.) La academia va al mercado - relaciones de científicos con clientes externos. Caracas: Fondo Editorial Fintec, 1995. p. 320

MOWERY, D.C.e ROSENBERG, N. "The U.S. national innovation system". In: Nelson, R.R. National innovations systems. Nova York, Oxford University Press, 1993, pp. 29-75.

NELSON, R.R. e ROSENBERG, N. "Technical innovation and national system". In: Nelson, R.R. (org.). National Innovation Systems. Nova York, Oxford University Press, 1993, pp.3-21.

NSF. "Science and Engineering Indicators". Washington D.C, National Science Board, National Science Foundation, 1993.

NSF - "Science and Engineering Indicators". Washington, D.C: National Science Board, National Science Foundation, 1996.

OECD. "Technology, public policy and the changing role of government laboratories". Paris, OECD, 1989.

OECD, "Organization for Economic Cooperation and Development, Technology and the Economy - The Key Relationships" (co-ordination and final preparation by F. Chesnais), Publications service, OECD, Paris, France, 1992.

PERRE, Gilberto. "Las relaciones de un grupo de físicos y de engenieros de São Carlos con el setor productivo". In: Vessuri, Hebe (org.). La academia va al mercado - Relaciones de científicos con clientes externos. Caracas, Fondo Editorial Fintec, 1995, pp. 263-291.

PETERS, L. Academic crossroads: The U.S. experience. Nova York, RPI Center For Technology Policy, Outubro 1987.

PLONSKI, G.A. "Novas alianças e parcerias em ciência, tecnologia e engenharia: A cooperação universidade-indústria". In: XV Simpósio Nacional de Pesquisa em Administração em Ciência e Tecnologia. Anais. São Paulo, 1990, 9p.

. "Cooperação universidade-empresa na Ibero-América: Estágio atual e perspectivas". In: Revista de Administração no 2. Abril/jun. 1995, vol. 30, pp. 65-74.

PLONSKI, G.A. (ed) Cooperacion empresa-universidad en Iberoamérica: avances recientes. São Paulo: Cyted, 1995a. 114p. 
QUINTAS, P. et al. "Academic-industry links and innovation: Questionning the science park model”. In: Technovation no 3. 1992, vol.12, pp. 161-175.

QUIRINO, Tarcízio R. "O programa de soja na Universidade Federal de Viçosa”. Cadernos de Gestão Tecnológica 7. São Paulo, Cyted, NPGCT/ USP, dezembro/1993.

RACHID, Alessandra. El Departamento de Ingeniería de Materiales (Dema) de la UFSCar. In: Vessuri, Hebe (org.). La academia va al mercado - relaciones de científicos con clientes externos. Caracas, Fondo Editorial Fintec, 1995.

SAGASTI, F. Science and technology for development. Otawa, Canadá: IDRC, 1980, 56 p..

SBRAGIA, Roberto \& TERRA, José C. C. - "Embraer: Trajetória de uma empresa de alta tecnologia brasileira". Cadernos de Gestão Tecnológica, 8. São Paulo: Cyted.

STEFANUTO, Giancarlo N. "Los límites del éxito de la investigación académica en el ambiente industrial”. In: Vessuri, Hebe (org.). La academia va al mercado - Relaciones de científicos con clientes externos. Caracas, Fondo Editorial Fintec, 1995. pp. 137-162.

SUTZ, J. Universidad y sectores productivos. Buenos Aires, Centro Editor da América Latina, 1994, 97p.

VELHO, S. Relações universidade-empresa: Desvelando mitos”. Brasília, junho de 1995 (cópia tipográfica).

VESSURI, H.M.C. "La ciencia académica em América Latina en el siglo XX". In: Revista de Estudios Sociales de la Ciencia (Redes) ํㅜ. 2. Buenos Aires, Dezembro 1994, vol. 1, pp. 41-76.

WEBSTER, A.J. "International evaluation of academic-industry relations: Contexts and analysis". In: Science and Public Policy no 2. Abril 1994a. vol. 21, pp. 72-78.

. "Bridging institutions: The role of contract research organizations in technology transfer". Science and Public Policy nํ 2. Abril 1994b, vol. 21, pp. 89-97.

WEBSTER, A.J. e ETZKOWITZ, H. Academic-industry relations: the second academic revolution? Londres, Science Policy Support Group, 1991, 31p. (SPSG concept paper ํㅜㄹ). 


\section{ARTICLES}

Qualification and productive restructuring:

The state of art of researches on education

Eneida Oto Shiroma e Roselane Fátima Campos.

Skills and restructuring of industrial relations:

A new bargaining instrument?

Nadya Araújo Castro.

Productive restructuring in the automotive industry in São Paulo:

Worker's perceptions of changes in work condition

Maria Rosa Lombardi.

Women's work in the clothing industry: a case study

Raquel da Silva Gazzana..

Productive Restructuring in bankins sector:

The reality of the 1990's

Sônia M. G. Larangeira...

Considerations about the trade sector:

Profile restructuring and trends

Marilis Lemos de Almeida.

Redesigning Institutional Networks: Inter-Firm Relationship,

Work and Education in the White Goods Industrial Sector

Leda Gitahy/ Adriana Marques da Cunha e Alessandra Rachid.

The universty-industry-govermnment relationship:

A study of the State University of Campinas (Unicamp)

Sandra Brisolla/Solange Corder/Erasmos Gomes e Débora Mello.

Contents. 